\title{
Time-Packing as Enabler of Optical Feeder Link Adaptation in High Throughput Satellite Systems
}

\author{
Joan Bas, Senior Member, IEEE, and Alexis A. Dowhuszko, Senior Member, IEEE \\ Centre Tecnològic de Telecomunicacions de Catalunya (CTTC/CERCA), Castelldefels (Barcelona), Spain \\ Email: joan.bas@cttc.es ; alexis.dowhuszko@cttc.es
}

\begin{abstract}
This paper carries out a theoretical study of the data rate that a High Throughput Satellite (HTS) system with fullyregenerative payload may achieve when using an intensity modulation/direct detection optical feeder link. A low-order $M$-ary Pulse Amplitude Modulation (M-PAM) with time-packing is used to modulate the intensity of the laser diode beam, making use of an external Mach-Zehnder modulator. These $M$-PAM symbols are recovered in the satellite with the aid of a photodetector, and are then encapsulated into the $5 \mathrm{G}$ radio frame of the access link. The modulation order and the overlapping factor of the uncoded transmission are jointly selected to tackle the impact that slowly-varying weather conditions introduce. Moreover, the intersymbol interference that time-packing introduces is mitigated in reception using a Viterbi equalizer. As expected, time-packing enables a finer link adaptation granularity than the one that adaptive modulation can provide on an optical feeder link without coding, enabling to adjust the spectral efficiency according to slowly-varying attenuation that thin cloud layers introduce.
\end{abstract}

Index Terms-High throughput satellite; optical feeder link; regenerative payload; link adaptation; time-packing.

\section{INTRODUCTION}

High Throughput Satellite (HTS) systems are evolving to enable $5 \mathrm{G}$ data services in those remote areas of the globe in which terrestrial mobile access is not available [1]. To support an aggregate data rate of few Terabit-per-second (Tbps), the GEO satellite should utilize a very large number of spotbeams with an aggressive frequency reuse factor in the radio access link (i.e., from the satellite to the user terminals) [2], and possibly optical wireless technology to support a similar point-to-point data rate on the feeder link (i.e., from the gateway to the satellite) [3]. Though there are few approaches to implement the optical feeder link [4], in this paper we focus on the fully regenerative payload case, in which the optical feeder link terminates in the satellite, and the $5 \mathrm{G}$ radio frame is generated on-board the satellite [5]. This way, realvalued modulation can be used to transport the payload bits on the optical feeder link, which is compatible with an Intensity Modulation (IM)/Direct Detection (DD) implementation.

GEO satellites are located over the equator at a height of about $36000 \mathrm{~km}$, implying a free-space path loss in the order of $290 \mathrm{~dB}$. To close such a demanding link budget, high-power transmitters and high-sensitivity receivers with Erbium-Doped Fibre Amplifiers (EDFA) are needed. In addition, optical telescopes with gains in the order of $100 \mathrm{dBi}$ should be used in

This work has received funding from the Ministry of Science, Innovation and Universities of Spain under project TERESA-TEC2017-90093C3-1-R (AEI/FEDER,UE) and from the Catalan Government under Grants 2017-SGR-00891 and 2017-SGR-01479. both extremes of the optical feeder link [6]. Finally, the impact that bad weather conditions have on the received optical signal must be also considered. The power loss that turbulenceinduced fading introduces can be reduced to just few dBs by using multiple optical apertures placed in the same satellite gateway (micro-diversity). However, the only way to tackle the absorption that slowly-moving clouds introduce is by reserving a margin of few-tens-of-dB in the link budget, which implies a waste of resources in presence of clear skies. Unfortunately, since real-valued modulations such as $M$-PAM are used in the IM/DD optical feeder link, there are limited degrees-offreedom for link adaptation, as the BER of $M$-PAM grows notably when $M \gg 2$. Therefore, if time-packing is added on top of the adaptive modulation scheme, the overlapping factor of the $M$-PAM signal can be used as an additional parameter to adapt the spectral efficiency of the optical feeder link.

Faster-than-Nyquist signaling, also known as time-packing, was proposed in the 1970 s as a simple solution to increase the spectral efficiency of a communication channel [7]. More precisely, it was shown that in presence of a binary sequence of Sinc-pulses, a 25\%-data-rate-increase is achievable by shrinking the time between adjacent Sinc-pulses to about $80.2 \%$ of the Nyquist symbol time. By doing so, the minimum Euclidean distance between received constellation points did not change, enabling a higher data rate without augmenting the communication bandwidth. With the implementation of timepacking, the use of high-order modulations could be avoided; therefore, this signaling is specially appealing for satellite systems with optical feeder link, as the Peak-to-Average Power Ratio (PAPR) of the transmit signal does not grow notably, and thus the power of the non-linear distortion and the signaldependent noise that are added can be kept low [8], [9].

In this paper, we focus on a HTS system with fullyregenerative payload, where the optical feeder link is prepared to tackle the moderate cloud attenuation that slowly-varying weather conditions introduce. In this situation, adaptive modulation with time-packing signaling can be used in transmission to maximize the spectral efficiency of the optical feeder link, and the Inter-Symbol Interference (ISI) that time-packing introduces can be mitigated in reception with the aid of a Viterbi equalizer. Different design parameters are considered in the performance evaluation, such as the overlapping factor, the roll-off factor of the Square-Root Raised-Cosine (SRRC) filters, and the number of states of the Viterbi equalizer that detects the sequence of received symbols on-board the satellite. As expected, the use of time-packing provides additional 
degrees-of-freedom to implement the adaptation of the optical feeder link, enabling a higher spectral efficiency than the one obtained with adaptive modulation solely. Though error control coding can be added on top of the adaptive modulation with time-packing optical transmission, its performance evaluation is not considered in this paper for the sake of brevity.

The rest of the paper is organized as follows: Section II presents the principles of time-packing and the details of the Viterbi equalizer that needs to be placed in reception. Section III introduces the system model and describes the most important blocks of the optical feeder link, including the IM transmitter, the optical wireless channel, and the DD receiver. The details of the simulation setup, as well as the evaluation of adaptive modulation with time-packing signaling are presented in Section IV. Finally, conclusions are drawn in Section V.

\section{THEORETICAL BACKGROUND}

This section summarizes the key theoretical principles to be taken into account when adding time-packing into the realvalued modulation scheme of our IM/DD optical feeder link.

\section{A. Principle of time-packing}

The continuous-time signal that a transmitter with timepacking generates can be written as

$$
s(t)=\sum_{k} s[k] g_{\mathrm{tx}}\left[t-k(1-\delta) T_{\mathrm{s}}\right],
$$

where $k$ is the position of the data symbol in the input stream $\{s[k]: k=1, \ldots\}, T_{\mathrm{s}}$ is the Nyquist symbol time, $g_{\mathrm{tx}}(t)$ is the time response of the transmit pulse-shaping filter, and $\delta$ is the overlapping factor used for time-packing. As in conventional communication systems, transmit pulses with response $g_{\mathrm{tx}}(t)$ have unit energy and are orthogonal when shifted by integer multiples of $T_{\mathrm{s}}$. However, it is important to note that when using time-packing, this orthogonality property is lost as $\int_{-\infty}^{\infty} g_{\mathrm{tx}}(t) g_{\mathrm{tx}}\left(t-n(1-\delta) T_{\mathrm{s}}\right) d t \neq 0$ usually holds. Therefore, Inter-Symbol Interference (ISI) is added but, in return, a timepacking signaling rate $R_{\mathrm{s}}^{\prime}=R_{\mathrm{s}} /(1-\delta) \geq R_{\mathrm{s}}=1 / T_{\mathrm{s}}$ can be achieved without increasing the communication bandwidth. In brief, time-packing enables a more efficient use of the communication bandwidth at the expenses of adding ISI, which must be mitigated in reception using advanced detectors.

Let us assume that the continuous-time received signal is $r(t)=s(t)+n(t)$, where $n(t)$ is Additive White Gaussian Noise (AWGN). The sufficient statistics for symbol detection is obtained after applying Matched Filtering (MF) [10], i.e.,

$$
\begin{aligned}
r[n] & =\int_{-\infty}^{\infty} r(t) g_{\mathrm{rx}}\left(t-n(1-\delta) T_{\mathrm{s}}\right) d t \\
& =\sum_{k} s[k] c[k-n]+\eta[n],
\end{aligned}
$$

where $g_{\mathrm{rx}}(t)=g_{\mathrm{tx}}(-t)^{*}$ and, due to that,

$$
\begin{aligned}
c[k-n] & =\int_{-\infty}^{\infty} g_{\mathrm{tx}}\left(t-k(1-\delta) T_{\mathrm{s}}\right) g_{\mathrm{tx}}\left(-t+n(1-\delta) T_{\mathrm{s}}\right)^{*} d t \\
\eta[n] & =\int_{-\infty}^{\infty} n(t) g_{\mathrm{tx}}\left(-t+n(1-\delta) T_{\mathrm{s}}\right)^{*} d t
\end{aligned}
$$

which is the so-called Ungerboeck observation model [11]. However, this model is not practical since the ISI in (2) is
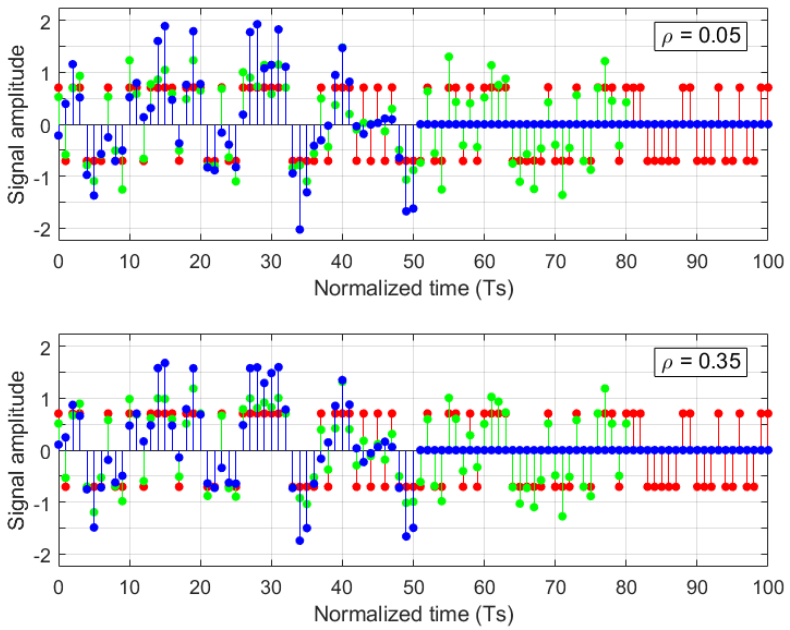

Fig. 1: Received signal samples at MF output assuming that 100 symbols with 2-PAM and SRRC filtering are transmitted with time-packing. Upper panel: $\rho=0.05$ (small roll-off factor). Lower panel: $\rho=0.35$ (large rolloff factor). Red stems: $\delta=0 \%$ (no overlapping). Green stems: $\delta=20 \%$ (moderate overlapping). Blue stems: $\delta=50 \%$ (strong overlapping).

non-causal and the noise samples $\eta[n]$ are correlated. In order to circumvent these problems, the signal after MF is passed through a whitening filter [12], which provides

$$
r^{\prime}[n]=\sum_{k} s[k] c^{\prime}[k-n]+\eta^{\prime}[n],
$$

where $c^{\prime}[k]$ for $k \neq n$ is causal ISI verifying $c^{\prime}[k] * c^{\prime}[-k]^{*}=$ $c[k]$, and $\eta^{\prime}[n]$ are AWGN samples. This ISI will depend on the modulation, roll-off, and overlapping factor that are used.

To illustrate this concept, Fig. 1 shows the received samples of a sequence of 2-PAM symbols when using SRRC filters with different roll-off factors $\rho$ and overlapping factors $\delta$. Note that each isolated SRRC pulse spans in time between $-50 T_{\mathrm{S}}$ and $50 T_{s}$. As expected, when the overlapping factor $\delta$ grows, the duration of the received signal sequence is reduced at the expense of increasing its amplitude variability or, equivalently, the PAPR. Concerning the roll-off factor, it is possible to show that as $\rho$ grows, the energy of the SRRC pulse spreads less and less in time and, consequently, the PAPR is slightly reduced.

Fig. 2 shows the PAPR of the transmit signal $s(t)$ when using $M$-PAM $(M=2,4,8)$ after being sampled at $t=k T_{\mathrm{s}}$ for $k=0, \ldots,\lfloor(1-\delta) 100\rfloor$, assuming that both $\delta$ and $\rho$ take different values. As previously mentioned, when comparing the three sub-figures, it is possible to observe that the PAPR grows slightly as $\rho$ decreases. Furthermore, when studying the effect of $\delta$ in the PAPR, it is possible to see that in all curves there is an initial part in which the PAPR decreases and, after that, it starts to constantly grow. Leaving aside this low-overlapping-factor region, it is possible to conclude that the overall tendency is that both ISI and PAPR grow as $\delta$ is increased and $\rho$ is decreased [13]. Then, if Maximum-Likelihood Sequence Estimation (MLSE) is implemented in reception, the impact of ISI could be mitigated, and the spectral efficiency of the modulation scheme with time-packing will be better than the one with baseline signaling an no time-packing $(\delta=0)$. 


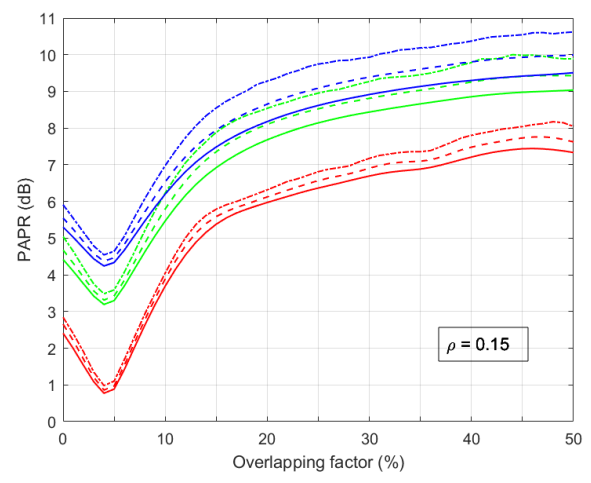

(a) Small roll-off factor $(\rho=0.15)$

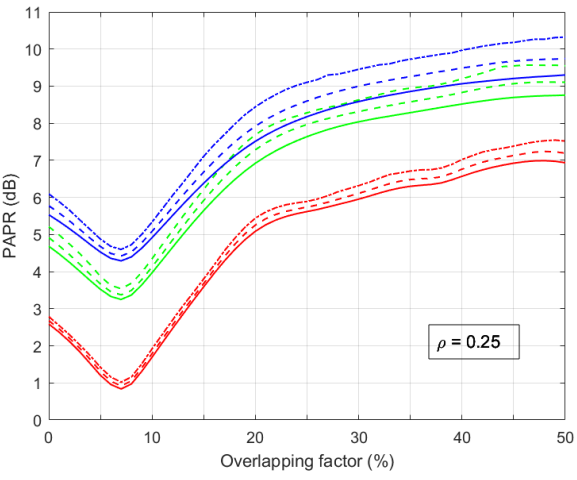

(b) Medium roll-off factor $(\rho=0.25)$

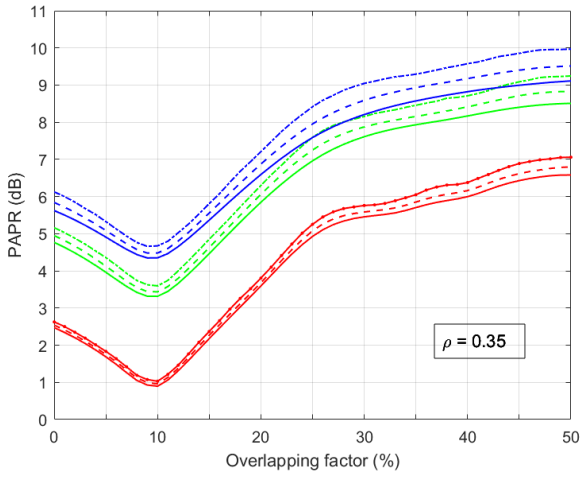

(c) Large roll-off factor $(\rho=0.35)$

Fig. 2: PAPR as function of overlapping factor $(\delta)$ for different $M$-PAM signaling and roll-off factors $(\rho)$. Modulations: 2-PAM (red lines), 4-PAM (green lines), and 8-PAM (blue lines). Solid lines: 90th-percentile of PAPR. Dashed lines: 99th-percentile of PAPR. Dashed-dotted lines: 99.99th-percentile of PAPR.

\section{B. MLSE detection for time-packing signaling}

Time-packing increases the data rate of a communication channel with constant bandwidth but, in return, introduces ISI that should be mitigated in reception to keep the Bit Error Rate (BER) low. When the minimization of the BER is the target goal, it is well-known that the optimal detector is the MLSE [10]. However, when looking for a trade-off between BER performance and implementation complexity, Maximum Likelihood (ML) detection methods assisted by channel shortening become more convenient [14], [15]. For this purpose, the selection of the roll-off factor of the SRRC filters $(\rho)$, the overlapping factor of the time packing signaling $(\delta)$, and the order of the $M$-PAM modulation should be done jointly, in order to keep the spectral efficiency of the communication channel as high as possible.

The ML estimation of sequence $\mathbf{s}=\left\{s_{0}, s_{1}, \ldots, s_{N-1}\right\}$ based on the received signal samples $r[n]$ attains the form

$$
\widehat{\mathbf{s}}=\arg \max _{\mathbf{s}} f(\mathbf{r} \mid \mathbf{s}) \text {, }
$$

where $f(\mathbf{r} \mid \mathbf{s})$ is the conditional Probability Density Function (PDF) of received signal samples $\mathbf{r}=\{r[n]: n=0, \ldots\}$ when $\mathbf{s}$ has been transmitted. Then, when $M$-PAM is used, $M^{N}$ trial sequences are obtained in total, which makes the computational complexity of the detector to grow as $\mathcal{O}\left(M^{N}\right)$.

This huge load can be notably reduced with the aid of the Viterbi algorithm [10], which takes advantage of the trellis structure of the overall communication channel (including the SRRC filters). In this case, the implementation complexity reduces from $\mathcal{O}\left(M^{N}\right)$ to $\mathcal{O}\left(M^{L_{\mathrm{T}}}\right)$, where $L_{\mathrm{T}}$ represents the channel memory. Fortunately, the overall channel memory with time-packing is a design parameter that can be controlled using channel shortening techniques [14], increasing the rolloff factor of the SRRC filters, and reducing the overlapping factor [12]. With these modifications, the PAPR in transmission and the energy consumption in reception are reduced but, in return, the spectral efficiency of the communication channel may be impacted if the BER due to residual ISI grows [13].

In this regard, Fig. 3 illustrates the number of $M$-PAM symbols or channel coefficients $L$, whose time-packing induced ISI must be mitigated to be within the target SNR gap $\Delta \mathrm{SNR}=3 \mathrm{~dB}$, with respect to the baseline $M$-PAM signaling without time-packing when BER $=10^{-3}$. Studying these figures, it is possible to conclude the number of channel coefficients introducing notable ISI grows with the order of the $M$-PAM modulation. For example, when $\rho=0.25$ and $\delta=0.30$, the number of coefficients with notable ISI are $L_{\mathrm{T}}=3$ for 2-PAM, $L_{\mathrm{T}}=4$ for 4-PAM, and $L_{\mathrm{T}}=5$ for 8-PAM. Unfortunately, it is not always practical to dimension the Viterbi equalizer for these $L_{\mathrm{T}}$ implementation parameters, particularly in presence of high-order $M$-PAM modulations.

The aim is to achieve a quasi-optimal performance only in the target SNR region of interest, in which the given $M$-PAM with time-packing signaling would be used. Therefore, we now study the combinations of roll-off factor $\rho$ and overlapping factor $\delta$ in which ML-decoding has an acceptable implementation complexity. It is well known that, as channel memory that is considered for ML-decoding grows, the BER tends to the one achieved with the baseline $M$-PAM signaling without time-packing (i.e., when the ISI power is null). However, our aim is to consider in the ML-decoding only part of the timepacking ISI; due to that, there will be some residual ISI that will make the received Signal-to-Interference-plus-Noise Ratio

$$
\operatorname{SINR}=\frac{\mathbb{E}\left\{|s[n]|^{2}\right\} c^{\prime}[0]^{2}}{\mathbb{E}\left\{|s[n]|^{2}\right\} \sum_{k=L_{\mathrm{T}}}^{\infty}\left|c^{\prime}[k]\right|^{2}+N_{0}},
$$

where $L_{T}$ is the number of ISI symbols considered in the Viterbi Algorithm, $N_{0}$ is the noise power, and $\mathbb{E}\left\{|s[n]|^{2}\right\}$ is the mean energy per $M$-PAM symbol. By replacing the SNR with the SINR, the following formula for the error probability of the $k$-th bit of the $M$-PAM symbol is obtained [16]:

$$
\begin{aligned}
P_{\mathrm{b}}[k] & =\frac{1}{M} \sum_{l=0}^{(M-1)-M 2^{-k}}\left\{( - 1 ) ^ { \lfloor \frac { l 2 ^ { k - 1 } } { M } \rfloor } \left(2^{l-1}\right.\right. \\
& \left.\left.-\left\lfloor\frac{l 2^{k-1}}{M}+\frac{1}{2}\right\rfloor\right) \operatorname{erfc}\left((2 l+1) \sqrt{\frac{3 \text { SINR }}{\left(M^{2}-1\right)}}\right)\right\},
\end{aligned}
$$

where $\lfloor x\rfloor$ is the greatest integer less than or equal to $x$ and $\operatorname{erfc}(x)=1 / \sqrt{2 \pi} \int_{x}^{\infty} \exp \left(-t^{2}\right) d t$ is the complementary error function. Then, it is possible to show that for $M$-PAM,

$$
\operatorname{BER}(M)=\sum_{k=1}^{m} \frac{P_{\mathrm{b}}[k]}{m}, \quad m=\log _{2}(M) .
$$




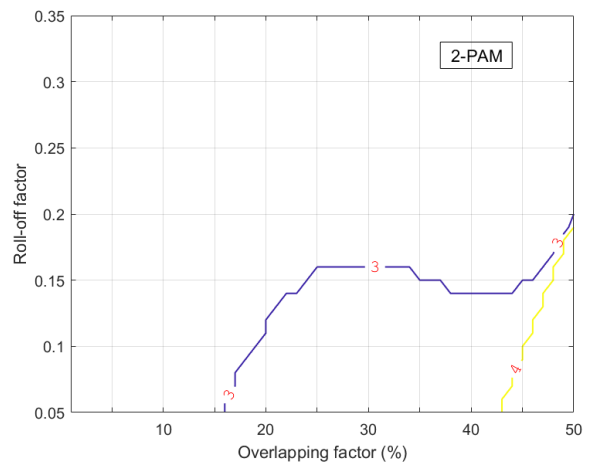

(a) Low-order modulation (2-PAM)

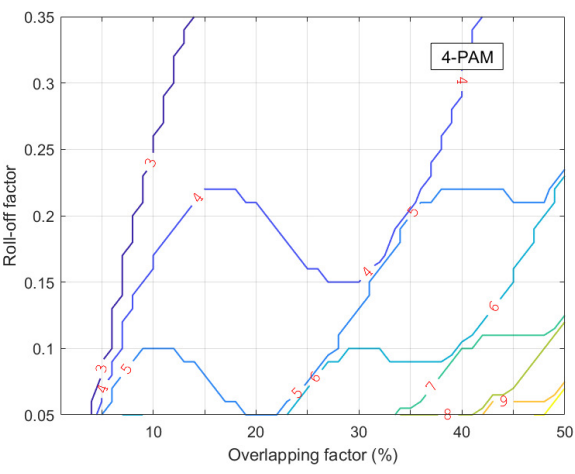

(b) Medium-order modulation (4-PAM)

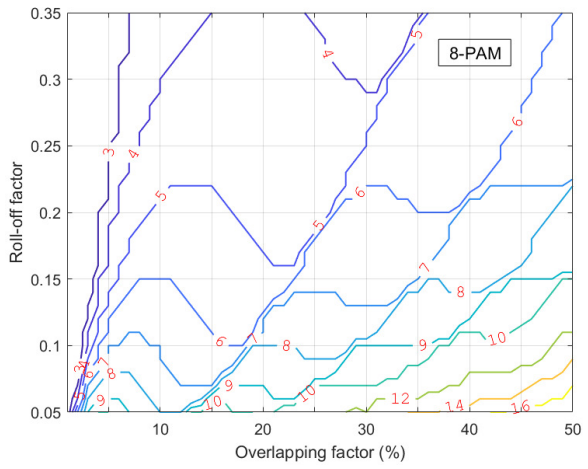

(c) High-order modulation (8-PAM)

Fig. 3: Number of adjacent $M$-PAM symbols (channel memory) in the Trellis of the Viterbi algorithm to verify the target SNR gap ( $\Delta$ SNR $=3.0 \mathrm{~dB}$ ) for different overlapping factors $(\delta)$ and roll-off factors $(\rho)$. Reference point: SNR to obtain BER $=10^{-3}$ using $M$-PAM signaling without time-packing.

The mutual information for $M$-PAM with time-packing signaling attains the form [17]

$$
\mathcal{I}_{\text {M-PAM }}(s[n] ; r[n])=\mathcal{H}(r[n])-\mathcal{H}(r[n] \mid s[n]),
$$

where $\mathcal{H}(r[n])$ is the entropy of the received signal samples and $H(r[n] \mid s[n])$ represents the entropy of the received signal samples conditioned to the transmitted symbol. When the residual-ISI-plus-Noise can be approximated as Gaussian distributed, then $\mathcal{H}(r[n] \mid s[n])=\frac{1}{2} \log _{2}\left(2 \pi \mathrm{e} P_{N_{\mathrm{T}}}\right)$, with $P_{N_{\mathrm{T}}}=$ $\mathbb{E}\left\{|s[n]|^{2}\right\} \sum_{k=L_{\mathrm{T}}}^{\infty}\left|c^{\prime}(k)\right|^{2}+N_{0}$. Similarly, according to [17],

$$
\mathcal{H}(r[n])=-\frac{1}{N_{\mathrm{R}}} \sum_{k=1}^{N_{\mathrm{R}}} \log _{2}\left(\sum_{l=0}^{M-1} \frac{\exp \left(-\frac{|s[k]+w[k]-s[l]|^{2}}{P_{N_{\mathrm{T}}}}\right)}{\sqrt{2 \pi P_{N_{\mathrm{T}}}}}\right),
$$

being $N_{\mathrm{R}}$ the number of Monte-Carlo runs. Finally, the capacity for $M$-PAM with time-packing signaling is given by

$$
\mathcal{C}_{\text {M-PAM }}(\rho, \delta)=\frac{1}{(1-\delta)(1+\rho)} \max _{f(s[l])}\left\{\mathcal{I}_{\text {M-PAM }}\right\},
$$

assuming that the penalization for bandwidth re-growth for using non-Sinc pulse-shaping filters is considered. Based on these previous formulas, the feasible throughput becomes

$$
\operatorname{TH}(\rho, \delta)=W \frac{(1-\mathrm{BLER}) \log _{2}(M) R_{\mathrm{c}}}{(1-\delta)(1+\rho)}, \quad W=\frac{1}{2 T_{\mathrm{s}}},
$$

where the Block Length Error Rate (BLER) is computed encapsulating $L_{\mathrm{p}}$ bits per packet, i.e., BLER $=1-(1-\mathrm{BER})^{L_{\mathrm{p}}}$, and the error control coding rate $R_{\mathrm{c}}=1$ for simplicity (uncoded case). The gain that error control coding (i.e., $R_{\mathrm{c}}<1$ ) provides on top of the performance of the uncoded optical feeder link with time-packed $M$-PAM signaling will be studied in detail in a future publication. Based on these theoretical principles, we are now ready to study the performance when time-packet $M$-PAM is used in an IM/DD optical feeder link.

\section{Optical FeEder Link With Time-Packing}

The simplified system model that corresponds to the optical feeder link of a HTS system with fully regenerative payload is illustrated in Fig. 4. It consists of a $M$-ary modulator that constructs a real-valued time domain signal, which is used to perform the IM of the Laser Diode (LD) light beam. In practice this is obtained with the aid of an external MachZehnder Modulator (MZM) working at a quadrature bias point.

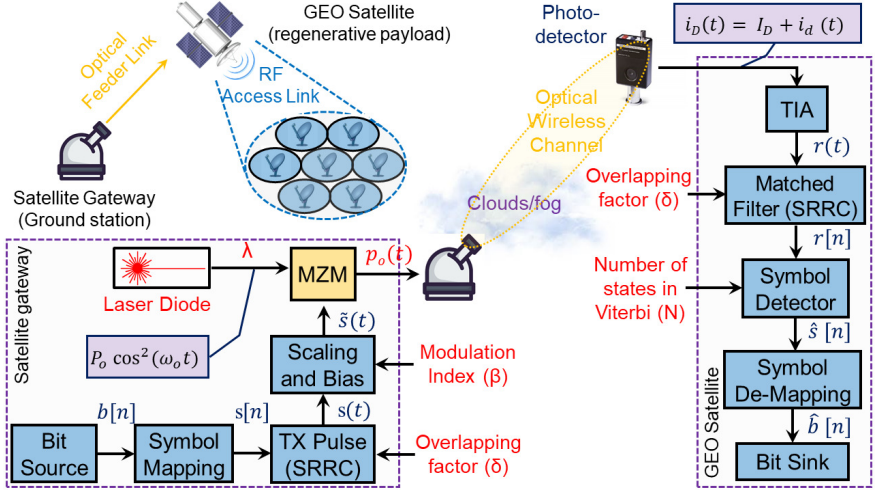

Fig. 4: Block diagram of a HTS system with regenerative payload. Blue blocks identify digital signal processing, whereas orange blocks identify the optical feeder link. Time-packing with overlapping factor $\delta$ is used to tackle slowlyvarying power loss that thin clouds/fog introduce (link adaptation).

At the receiver side, a Photodetector (PD) is used for the DD of the optical signal that reaches the GEO satellite and, after that, digital signal processing is applied to recover the stream of bits that was transmitted. With this payload data, the radio frame of the access link (e.g., DVB-S2X standard) is finally constructed on-board the satellite and transmitted to the user terminal on the Radio Frequency band that corresponds.

\section{A. Intensity modulation of optical carrier at ground station}

The continuous-time signal that modulates the intensity of the LD beam was presented in (1). Then, the driving voltage of the external MZM is given by

$$
v_{\mathrm{mzm}}(t)=V_{\mathrm{B}}+\beta \widetilde{s}(t)\left(V_{\pi} / \pi\right),
$$

where $V_{\mathrm{B}}$ and $V_{\pi}$ are the bias and half-wavelength voltages of the MZM, $\beta$ is the intensity modulation index, and

$$
\widetilde{s}(t)=s(t) / \sqrt{\mathbb{E}\left\{|s(t)|^{2}\right\}},
$$

is the normalized continuous-time $M$-PAM signal with unitary Root Mean Square (RMS) amplitude. We note that the specific choice of $\beta$ controls the range in which the MZM regularly works. Deep intensity modulation indexes (i.e., large $\beta$ ) increase the power on the optical feeder link sidebands but, at the same time, increment the non-linear distortion power on the electrical signal that is recovered on-board the satellite. 
The relation between the driving voltage $v_{\text {mzm }}(t)$ and the optical field at the MZM output $E_{\mathrm{o}}(t)$ is given by [18]

$$
E_{\mathrm{o}}(t)=\cos \left[\frac{\pi}{2} \frac{v_{\mathrm{mzm}}(t)}{V_{\pi}}\right] \sqrt{2 P_{\mathrm{o}, \mathrm{ld}}} \cos \left(\omega_{\mathrm{o}} t\right),
$$

where $P_{\mathrm{o}, \mathrm{ld}}$ is the mean optical power of the LD that feeds the MZM and $\omega_{\mathrm{o}}$ is the angular frequency of the unmodulated optical carrier that is transmitted with the optical sidebands. Then, the instantaneous value that the optical intensity modulated signal takes at the output of the MZM becomes

$$
\begin{aligned}
p_{\mathrm{o}}(t) & =E_{\mathrm{o}}^{2}(t)=\cos ^{2}\left(\frac{\pi}{2} \frac{v_{\mathrm{mzm}}(t)}{V_{\pi}}\right) 2 P_{\mathrm{o}, \mathrm{ld}} \cos ^{2}\left(\omega_{\mathrm{o}} t\right), \\
& =\left[1+\cos \left(\frac{\pi V_{\mathrm{B}}}{V_{\pi}}+\beta \widetilde{s}(t)\right)\right] P_{\mathrm{o}, \mathrm{ld}} \cos ^{2}\left(\omega_{\mathrm{o}} t\right) .
\end{aligned}
$$

When the quadrature bias point $V_{\mathrm{B}}=\left(3 V_{\pi}\right) / 2$ is used,

$$
\begin{aligned}
p_{\mathrm{o}}(t) & =[1+\sin (\beta \widetilde{s}(t))] P_{\mathrm{o}, \mathrm{ld}} \cos ^{2}\left(\omega_{\mathrm{o}} t\right) \\
& \approx \underbrace{[1+\beta \widetilde{s}(t)]}_{\text {modulating signal }} \underbrace{P_{\mathrm{o}, \mathrm{ld}} \cos ^{2}\left(\omega_{\mathrm{o}} t\right)}_{\text {optical carrier }} \quad \beta \ll 1,
\end{aligned}
$$

where the latter approximation is due to $\sin (x) \approx x$ for small $x$. In this situation, the effect that the MZM non-linear distortion has on the optical feeder link can be neglected.

\section{B. Optical wireless channel modeling}

The Free Space Loss (FSL) represents the largest power loss in the optical feeder link, and is given by

$$
L_{\mathrm{o}, \mathrm{fsl}}=\lambda^{2} /\left(4 \pi d_{\mathrm{fso}}\right)^{2},
$$

where $d_{\mathrm{fso}}$ is the link range and $\lambda$ is the wavelength that the optical feeder link utilizes. Besides the FSL, additional losses may be experienced when the optical signal propagates through the low layers of the atmosphere, particularly in case of bad weather conditions in which visibility is reduced. It is important to note that atmospheric losses $L_{\mathrm{o}}$,atm can be as low as few dBs in presence of Fog and Cirriform clouds, few tens of $\mathrm{dBs}$ in case of Stratocumulus and Altostratus, and from few hundreds to few thousand $\mathrm{dBs}$ in presense of Cumulonimbus. As expected, $L_{\mathrm{o}, \text { atm }}$ for cloudy weather will depend on the thickness and density of water droplets that clouds contain [19]. Though communication is not possible in presence heavy Cumuloninbus, our aim it to use time-packing as enabler of better link adaptation granularity, such that the throughput of the optical feeder link is maximized assuming partly cloudy weather in which $L_{\mathrm{o} \text {,atm }}$ is few tens of $\mathrm{dBs}$.

Finally, the atmospheric turbulence is caused by the mixing of warm and cold air on the different layers of the atmosphere. Turbulence generates small variations on the refractive index of the signal path, inducing a fluctuation on the received optical intensity that is known as Scintillation. In case of weak turbulence, the statistics of the received intensity modulated signal can be approximated with a Log-Normal distribution. Similarly, the exponential distribution can be used to model this turbulence-induced fading in case of strong turbulence, whereas the Gamma-Gamma distribution models this effect very well in a wider range of turbulence conditions. Although these stochastic models have been widely studied in the literature, transmit diversity techniques are needed to mitigate
TABLE I: Parameters of the optical feeder link of the HTS system.

\begin{tabular}{|c|l|c|c|}
\hline Symbol & Optical Link Parameter & Value & Unit \\
\hline$P_{\mathrm{o}, \mathrm{ld}}$ & Optical power of LD (incl. EDFA booster) & 47.0 & $\mathrm{dBm}$ \\
\hline$G_{\mathrm{o}, \mathrm{tx}}$ & Optical gain of transmitter (ground telescope) & 112.2 & $\mathrm{~dB}$ \\
\hline$G_{\mathrm{o}, \mathrm{rx}}$ & Optical gain of receiver (satellite telescope) & 114.1 & $\mathrm{~dB}$ \\
\hline$L_{\mathrm{o}, \mathrm{fs}}$ & FSL of optical link (1550 nm, 39000 km) & 290.0 & $\mathrm{~dB}$ \\
\hline$L_{\mathrm{o}, \mathrm{atm}}$ & Atmospheric loss due to thin cloud layers & $0-20$ & $\mathrm{~dB}$ \\
\hline$L_{\mathrm{o}, \mathrm{sys}}$ & System losses in the optical feeder link & 4.7 & $\mathrm{~dB}$ \\
\hline$G_{\mathrm{edfa}}$ & Gain of the optical amplifier (EDFA) & 50.0 & $\mathrm{~dB}$ \\
\hline$\mu$ & Responsivity of photodetector (PIN diode) & 0.5 & $\mathrm{~A} / \mathrm{W}$ \\
\hline$B_{\mathrm{e}}$ & Bandwidth of electrical filter (PD output) & 1.5 & $\mathrm{GHz}$ \\
\hline$B_{\mathrm{o}}$ & Bandwidth of optical channel (1550 nm) & 12.5 & $\mathrm{GHz}$ \\
\hline$\rho_{\mathrm{ase}}$ & PSD of amplified spontaneous emissions & $2.0 \times 10^{-19}$ & $\mathrm{~W} / \mathrm{Hz}$ \\
\hline$\rho_{\text {rin }}$ & PSD of RIN process (normalized) & -160 & $\mathrm{dBc} / \mathrm{Hz}$ \\
\hline$\rho_{\text {back }}$ & PSD of background noise at EDFA input & $7.6 \times 10^{-25}$ & $\mathrm{~W} / \mathrm{Hz}$ \\
\hline$i_{\mathrm{n}}$ & Electrical noise current spectral density & $1.0 \times 10^{-11}$ & $\mathrm{~A}$ \\
\hline$i_{\text {dark }}$ & Dark current at the PIN diode output & $1.0 \times 10^{-10}$ & $\mathrm{~A}$ \\
\hline
\end{tabular}

the impact of turbulence (e.g., multiple optical apertures). This is because the coherence time of the turbulence-induced fading states is much shorter than the propagation time of the optical signal from the ground station to the GEO satellite. To tackle this, few $\mathrm{dBs}$ of the optical feeder link budget are reserved as system losses to make the outage probability due to turbulence negligible. More detailed studies on the effect that turbulenceinduced fading has on the outage probability of the optical feeder link are left aside for a future publication.

\section{Direct Detection of the optical signal onboard the satellite}

The optical signal that illuminates the sensitive area of the $\mathrm{PD}$ in the satellite generates an electrical current that equals

$$
\begin{gathered}
i_{\mathrm{D}}(t)=I_{\mathrm{D}}+i_{\mathrm{d}}(t)=\mu \frac{G_{\mathrm{o}, \mathrm{tx}} G_{\mathrm{o}, \mathrm{rx}} G_{\mathrm{o}, \mathrm{edfa}}}{L_{\mathrm{o}, \mathrm{fs} \mathrm{l}} L_{\mathrm{o}, \mathrm{atm}} L_{\mathrm{o}, \mathrm{sys}}} \int_{t}^{t+T_{\mathrm{o}}} p_{\mathrm{o}}(\tau) d \tau, \\
\int_{t}^{t+T_{\mathrm{o}}} p_{\mathrm{o}}(\tau) d \tau=\frac{P_{\mathrm{o}, \mathrm{ld}}}{2}[1+\sin (\beta \widetilde{s}(t))]
\end{gathered}
$$

where $T_{\mathrm{o}}=2 \pi / \omega_{\mathrm{o}}$ is the period of the optical carrier, $\mu$ [A/W] is the PD responsivity, $G_{\mathrm{o}, \mathrm{tx}}$ and $G_{\mathrm{o}, \mathrm{rx}}$ are the optical gains of the transmit and receive telescopes, respectively, $G_{\mathrm{o} \text {,edfa }}$ is the gain of the Erbium-Doped Fiber Amplifier (EDFA) placed before the PD in satellite, and $L_{\mathrm{o} \text {,sys }}$ accounts the system losses in the optical feeder link. Note that the current in (20) can be divided into two terms, where the DC component is given by

$$
I_{\mathrm{D}}=\mathbb{E}\left\{i_{\mathrm{D}}(t)\right\}=\mu \frac{G_{\mathrm{o}, \mathrm{tx}} G_{\mathrm{o}, \mathrm{rx}} G_{\mathrm{o}, \mathrm{edfa}}}{L_{\mathrm{o}, \mathrm{fsl}} L_{\mathrm{o}, \mathrm{atm}} L_{\mathrm{o}, \mathrm{sys}}} \frac{P_{\mathrm{o}, \mathrm{ld}}}{2},
$$

and remains fixed regardless of $\beta$, whereas the $\mathrm{AC}$ component depends on the intensity modulation index and attains the form

$$
i_{\mathrm{d}}(t)=i_{\mathrm{D}}(t)-I_{\mathrm{D}}=I_{\mathrm{D}} \sin (\beta \widetilde{s}(t)) \approx I_{\mathrm{D}} \beta \widetilde{s}(t) \quad \beta \ll 1 .
$$

The SNR of the electrical signal that is direct-detected by the PD on-board the GEO satellite becomes

$$
\mathrm{SNR}_{\mathrm{e}, \mathrm{pd}}=\frac{\mathbb{E}\left\{\left|i_{\mathrm{d}}(t)\right|^{2}\right\}}{\mathbb{E}\left\{\left|n_{\mathrm{o}}(t)\right|^{2}\right\}} \approx \frac{I_{D}^{2} \beta^{2}}{\mathbb{E}\left\{\left|n_{\mathrm{o}}(t)\right|^{2}\right\}} \quad \beta \ll 1,
$$

where

$$
\begin{aligned}
\mathbb{E}\left\{\left|n_{\mathrm{o}}(t)\right|^{2}\right\} & =\mathbb{E}\left\{\left|i_{\text {shot }}(t)\right|^{2}\right\}+\mathbb{E}\left\{\left|i_{\text {thermal }}(t)\right|^{2}\right\} \\
& +\mathbb{E}\left\{\left|i_{\text {rin }}(t)\right|^{2}\right\}+\mathbb{E}\left\{\left|i_{\text {beat }}(t)\right|^{2}\right\},
\end{aligned}
$$




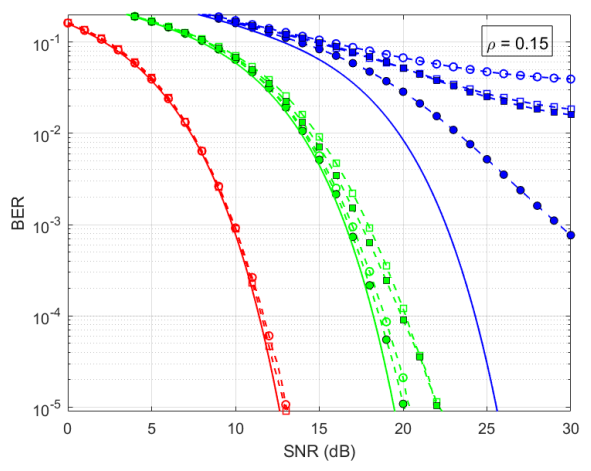

(a) Small roll-off factor $(\rho=0.15)$

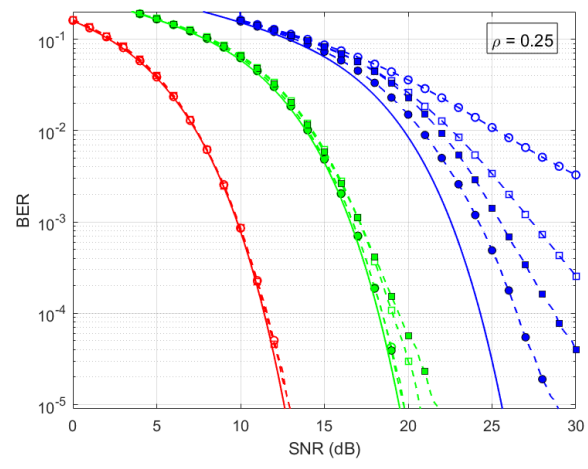

(b) Medium roll-off factor $(\rho=0.25)$

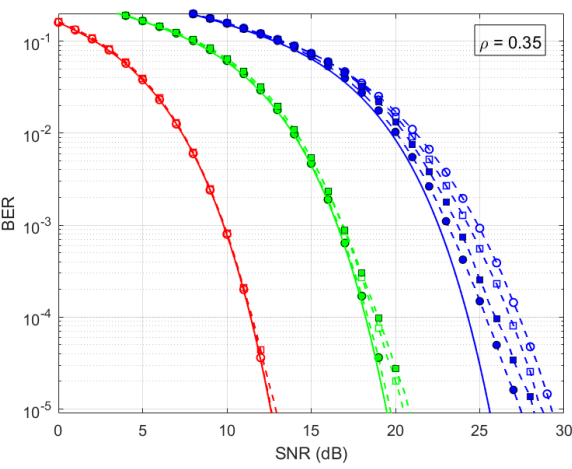

(c) Large roll-off factor $(\rho=0.35)$

Fig. 5: BER as function of the electrical SINR for different optical feeder link configurations. Modulation: 2-PAM (red lines), 4-PAM (green lines), and 8-PAM (blue lines). Overlapping factors: $\delta=0$ (solid lines), $\delta=0.15$ (dashed lines with circles), and $\delta=0.30$ (dashed lines with squares). Trellis states in Viterbi Algorithm: $N_{s}=1024$ for 2-/4-PAM and 512 for 8-PAM (unfilled markers) and $N_{s}=4096$ for 2-/4-/8-PAM (filled markers).

includes the contribution of all noise sources in the optical feeder link, namely the shot noise sources, thermal noise, Relative Intensity Noise (RIN) of LD, and beat noise [20]. Note that shot noise term includes the contribution of the received optical signal, the Amplified Spontaneous Emission (ASE) noise, background optical noise and the dark current noise, whereas the beat noise term accounts the effect of combining the received optical signal with the ASE noise.

When the received optical power is between -90 and $-20 \mathrm{dBW}$, it can be shown that the beat noise between received optical signal and ASE noise dominates the SNR performance of the optical feeder link [21]. In this situation, we have that

$$
\begin{aligned}
\mathbb{E}\left\{\left|n_{\mathrm{o}}(t)\right|^{2}\right\} & \approx \mathbb{E}\left\{\left|i_{\text {beat }}(t)\right|^{2}\right\}=i_{\text {sig-sp }}^{2}+i_{\text {sp-sp }}^{2} \\
& \approx i_{\text {sig-sp }}^{2}=4 I_{\mathrm{D}} I_{\text {ase }}\left(B_{\mathrm{e}} / B_{\mathrm{o}}\right),
\end{aligned}
$$

where $B_{\mathrm{o}}$ is the bandwidth of the optical signal at the input of the PD, which depends on the channel separation of the Wavelength-Division Multiplexing (WDM) technology that is used, $B_{\mathrm{e}}$ is the bandwidth of the electrical signal at the output of the PD, and $I_{\text {ase }}=\mu G_{\mathrm{o} \text {,edfa }} P_{\text {ase }}$ is the DC component generated by the ASE noise when $P_{\text {ase }}=\rho_{\text {ase }} B_{\mathrm{o}}$ is the equivalent noise power of the EDFA before amplification.

\section{Performance Evaluation}

Table I summarizes the parameters of the optical feeder link, including the optical gains and optical losses [22], and the different sources of optical noise [21]. Unless stated otherwise, the effect of any other parameter that is not listed in this table is assumed negligible (e.g., specific PD non-idealities and optical wireless channel impairments).

According to these values, mean received optical power is

$$
\begin{aligned}
& P_{\mathrm{o}, \mathrm{rx}}[\mathrm{dBm}]=P_{\mathrm{o}, \mathrm{ld}}[\mathrm{dBm}]+G_{\mathrm{o}, \mathrm{tx}}[\mathrm{dB}]+G_{\mathrm{o}, \mathrm{rx}}[\mathrm{dB}]-L_{\mathrm{o}, \mathrm{fss}}[\mathrm{dB}] \\
& \quad-L_{\mathrm{o}, \mathrm{sys}}[\mathrm{dB}]-L_{\mathrm{o}, \mathrm{atm}}[\mathrm{dB}]=-21.4[\mathrm{dBm}]-L_{\mathrm{o}, \mathrm{atm}}[\mathrm{dB}] .(27)
\end{aligned}
$$

In presence of clear-sky conditions (i.e., when $L_{\mathrm{o}, \mathrm{atm}}=0 \mathrm{~dB}$ ), the DC current generated by the optical signal at the output of the PD is $I_{\mathrm{D}}=181.11 \mathrm{~mA}$, whereas the DC current generated by the ASE noise is $I_{\text {ase }}=0.125 \mathrm{~mA}$ regardless the weather. Therefore, when the intensity modulation index $\beta=0.5$, the SNR of the electrical signal at the output of the PD becomes

$$
\mathrm{SNR}_{\mathrm{e}, \mathrm{pd}}[\mathrm{dB}]=28.78[\mathrm{~dB}]-L_{\mathrm{o}, \mathrm{atm}}[\mathrm{dB}] .
$$

Larger intensity modulation indexes $\beta$ could be used without increasing the MZM non-linear distortion notably, provided that Digital Pre-Distortion (DPD) compensation is implemented in transmission [20]. Though some DPD techniques could be as simple as optimizing the separation between $M$-ary constellation points to minimize non-linear distortion power, for the sake of simplicity, in this paper we assume that the dynamic range of the input signal is set low enough, such that the MZM works on its linear region most of the time.

Fig. 5 shows the BER as function of the received SINR for different $M$-PAM modulation schemes, roll-off factors $\rho$, overlapping factor $\delta$. Two implementation complexities for the ML decoder have been considered, namely $N_{\mathrm{s}}=512 / 1024$ states (moderate Trellis) and 4096 states (demanding Trellis). When comparing these curves, it is possible to see that in case of 2-PAM and 4-PAM, most of the roll-off factors and overlapping factors that have been evaluated provide a similar BER to the one attainable when time-packing is not used (lower bound in absence of ISI, when $\delta=0$ ). However, in case of 8-PAM, the BER performance degrades notably, particularly when $\rho=0.15$ (lowest roll-off factor), $\delta=0.3$ (highest overlapping factor), and $N_{\mathrm{s}}=512$ (moderate implementation complexity). This is because 8-PAM is the largest order modulation under evaluation and, due to that, the Trellis states are only enough to tackle the ISI generated by few adjacent symbols (i.e., short channel memory). Due to this, the residual ISI is high, and the BER performance is notably penalized. However, as the roll-off factor increases, the energy of SRRC pulses concentrates on fewer channel coefficients and, due to that, the BER performance is closer to the one attainable when the ISI due to time-packing is completely suppressed.

Finally, Fig. 6 illustrates the throughput as function of the cloud attenuation, normalized by the communication bandwidth, that $M$-PAM with time-packing is able to achieve when the roll-off factor is $\rho=0.25$. For each modulation scheme, three different overlapping factors are considered, namely: $\delta=0$ (no overlapping), $\delta=0.15$ (low overlapping), and $\delta=0.3$ (medium overlapping). Moreover, the number of bits that are encapsulated per data packet is $L_{\mathrm{p}}=2536$. 


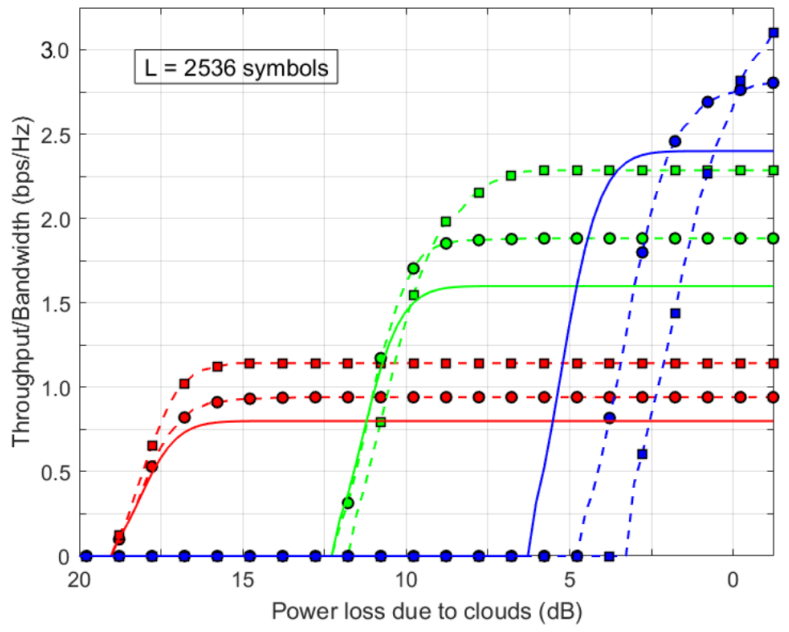

Fig. 6: Normalized throughput of the IM/DD optical feeder link as function of the cloud attenuation when using $M$-PAM with time-packing ( $\rho=0.25$, $N_{\mathrm{s}}=4096$ states, $L_{p}=2536$ bits). Modulation: 2-PAM (red lines), 4-PAM (green lines), 8-PAM (blue lines). Overlapping factor: $\delta=0$ (solid lines), $\delta=0.15$ (dashed-lines with circles), $\delta=0.3$ (dashed-lines with squares).

We note that this packet length is aligned with the maximum Transport Block Size (TBS) from the Physical Uplink Shared Channel (PUSCH) of Narrowband (NB)-IoT radio technology standard [23]. Based on this curve, it is possible to see that 2-PAM with $\delta=0.3$ is the most convenient signaling for a cloud attenuation between 19 and $11 \mathrm{~dB}$. However, as we reduce the cloud attenuation, then 4-PAM with different overlapping factors gives the best throughput for cloud attenuation between 11 and $4 \mathrm{~dB}$, whereas 8-PAM with different overlapping factors is the best choice when cloud attenuation is lower than $4 \mathrm{~dB}$. To sum up, the use of $M$-PAM with timepacking enables a finer granularity when designing the link adaptation mechanism of the optical feeder link.

\section{CONCLUSION}

In this paper, we made a theoretical analysis of the throughput that is achievable when $M$-PAM with time-packing is used to modulate in intensity the optical feeder link of a HTS system with fully-regenerative payload. The ISI that timepacking introduces was tackled on-board the GEO satellite, with the aid of a Viterbi equalizer that managed to mitigate the impact of ISI for most transmission schemes under analysis. Based on these results, it was possible to show that $M$-PAM with time-packing is a good solution improve the granularity of link adaptation for IM/DD optical links, where the use of real-valued modulations limits notably the available transmission schemes when error control coding is not used. Thanks to this approach, the slowly-varying attenuation that thin cloud layers introduce can be addressed by changing the modulation order $M$ and the overlapping factor $\delta$ of the waveform that modulates the intensity of the optical feeder link. Therefore, it is possible to conclude that time-packing signaling is an appealing solution to implementing the optical feeder link of HTS system, designed to provide global 5G/5G+ connectivity.

\section{REFERENCES}

[1] M. Jia, X. Gu, Q. Guo, W. Xiang, and N. Zhang, "Broadband hybrid satellite-terrestrial communication systems based on cognitive radio toward 5G," IEEE Wireless Commun., vol. 23, no. 6, pp. 96-106, Dec. 2016.

[2] D. Giggenbach, E. Lutz, J. Poliak, R. Mata-Calvo, and C. Fuchs, "A high-throughput satellite system for serving whole Europe with fast Internet service, employing optical feeder links," in Proc. ITG Symposium on Broadband Coverage in Germany, Apr. 2015, pp. 1-7.

[3] I. Ahmad, K. Nguyen, and N. Letzepis, "Performance analysis of high throughput satellite systems with optical feeder links," in Proc. IEEE Global Commun. Conf., Dec. 2017, pp. 1-7.

[4] R. Mata-Calvo, D. Giggenbach, A. Le Pera, J. Poliak, R. Barrios, and S. Dimitrov, "Optical feeder links for very high throughput satellitesSystem perspectives," in Proc. Ka Broadband Commun., Navigation and Earth Observation Conf., Oct. 2015, pp. 1-7.

[5] 3GPP, "Technical Specification Group Services and System Aspects: Study on using Satellite Access in 5G; Stage 1,' Tech. Rep. TS 22.822, 2018, rel. 16.

[6] N. Perlot, T. Dreischer, C. Weinert, and J. Perdigues, "Optical GEO feeder link design," in Future Netw. Mobile Summit, July 2012, pp. 1-8.

[7] J. Mazo, "Faster-than-Nyquist signaling,", Bell Syst. Tech. Journal, vol. 54, pp. 1451-1462, Oct. 1975.

[8] Y. Qiao, J. Zhou, M. Guo, X. Tang, J. Qi, and Y. Lu, "Fasterthan-Nyquist signaling for optical communications," in Proc. OptoElectronics and Commun. Conf., July 2018, pp. 1-2.

[9] J. Bas and A. Pérez-Neira, "On the physical layer security of IoT devices over satellite," in Proc. Europ. Sig. Proces. Conf., Sept. 2019, pp. 1-5.

[10] G. Forney, "Maximum-likelihood sequence estimation of digital sequences in the presence of intersymbol interference," IEEE Trans. Inform. Theory, vol. 18, no. 3, pp. 363-378, May 1972.

[11] G. Ungerboeck, "Adaptive maximum-likelihood receiver for carriermodulated data-transmission systems," IEEE Trans. Commun., vol. 22, no. 5, pp. 624-636, May 1974.

[12] F. Rusek and J. Anderson, "Constrained capacities for Faster-thanNyquist signaling," IEEE Trans. Inform. Theory, vol. 55, no. 2, pp. 764-775, Feb. 2009.

[13] T. Delamotte, A. Knopp, and G. Bauch, "Faster-than-Nyquist signaling for satellite communications: A PAPR analysis," in Proc. Int. ITG Conf. Systems, Commun and Coding, Feb. 2017, pp. 1-6.

[14] J. Fan, Y. Ren, Y. Zhang, and X. Luo, "MLSE equalizer with channel shortening for Faster-than-Nyquist signaling," IEEE Photonics Tech. Letters, vol. 30, no. 9, pp. 793-796, Mar. 2018.

[15] L. Chen, J. Liu, and C. Guo, "Training aided frequency domain equalization with maximum-likelihood sequence detection in coherent optical communications systems using FTN signaling," in Proc. Int. Conf. Optical Commun. Netw., July 2015, pp. 1-3.

[16] K. Cho and D. Yoon, "On the general BER expression of one- and twodimensional amplitude modulations," IEEE Trans. Commun., vol. 50, no. 7, pp. 1074-1080, Nov. 2002.

[17] D. Arnold, H. Loeliger, and P. V. et al., "Simulation-based computation of information rates for channels with memory," IEEE Trans. Inform. Theory, vol. 52, no. 8, pp. 3498-3508, July 2006.

[18] J. Leibrich, A. Ali, H. Paul, W. Rosenkranz, and K. Kammeyer, "Impact of modulator bias on the OSNR requirement of direct-detection optical OFDM," IEEE Photonics Tech. Letters, vol. 21, no. 15, pp. 1033-1035, Aug. 2009.

[19] J. Churnside and K. Shaik, "Atmospheric propagation issues relevant to optical communications," National Oceanic and Atmopheric Administration, Technical memo. Report No. NOAA TM ERL WPL 159, pp. 1-57, Jan. 1989.

[20] A. Dowhuszko, A. Mengali, P. Arapoglou, and A. Pérez-Neira, "Total degradation of a DVB-S2 satellite system with analog transparent optical feeder link," in Proc. IEEE Global Commun. Conf., Dec. 2019, pp. 1-6.

[21] A. Mengali, "Optical feeder link satellite systems," in Link optimization in future generation satellite systems. $\mathrm{PhD}$ Thesis, University of Luxembourg, Oct. 2018, ch. 4, pp. 61-86.

[22] B. Roy, S. Poulenard, S. Dimitrov, R. Barrios, D. Giggenbach, A. Kernec, and M. Sotom, "Optical feeder links for high throughput satellites," in Proc. IEEE Int. Conf. on Space Optical Systems and Applications, Oct. 2015, pp. 1-6.

[23] 3GPP, "Technical Specification Group Radio Access Network; Evolved Universal Terrestrial Radio Access (E-UTRA); Physical layer procedures," Tech. Rep. TS 36.213, 2020, rel. 16.1. 